\title{
NUMERICAL SIMULATION OF SHOCK BOUNDARY LAYER INTERACTION USING SHOCK FITTING TECHNIQUE
}

\author{
A. ASSONITIS ${ }^{1}$, R. PACIORRI ${ }^{2}$ AND A. BONFIGLIOLI ${ }^{3}$ \\ ${ }^{1}$ Università degli Studi di Roma "La Sapienza" \\ Via Eudossiana 18, 00184, Rome, Italy \\ alessia.assonitis@uniroma1.it \\ ${ }^{2}$ Università degli Studi di Roma "La Sapienza" \\ Via Eudossiana 18, 00184, Rome, Italy \\ renato.paciorri@uniroma1.it \\ ${ }^{3}$ Università degli Studi della Basilicata \\ Viale dell'Ateneo Lucano 10, 85100 Potenza, Italy \\ aldo.bonfiglioli@unibas.it
}

Key words: Shock-Fitting, Shock/Boundary-Layer Interaction

\begin{abstract}
The unstructured shock-fitting algorithm originally proposed in Ref. [3] has been further developed to make it capable of dealing with shock-wave/boundary-layer interactions (SWBLIs). This paper illustrates the algorithmic features of the technique and its application to 2D flow-configurations featuring different SWBLIs, including the transonic turbulent flow past a symmetrical airfoil and a laminar oblique-shock reflection.
\end{abstract}

\section{INTRODUCTION}

Accurate skin-friction and wall heating predictions of flows featuring SWBLIs require the numerical modeling of the shock-wave, a task which can be achieved using either shock-capturing (S-C) or shock-fitting (S-F). The S-C method is built upon the integral, conservation-law form of the governing equations: no special treatment is required for modeling the discontinuities, but their thickness depends on the local mesh-spacing, which may be orders of magnitude larger than the true (physical) shock-width. A different way to proceed is offered by the S-F approach: it consists in identifying and tracking the motion of the discontinuities, which are treated as internal boundaries of zero thickness which bound regions of the flow-field where a continuous solution of the governing PDE exists. In the S-F approach the speed of the discontinuity and its upstream and downstream states are computed by solving the Rankine-Hugoniot (R-H) jump relations, whereas the flow in the smooth regions can be approximated using a gas-dynamic solver. The S-F technique has been developed by Gino Moretti $[1,9]$ and his collaborators since the late 1960s. Its algorithmic complexity, partly related to the used of structured grids, contributed to the gradual dismissal of the technique: by the turn of the century nearly all high-speed flows were simulated using the S-C approach. Over the last decade, however, two of the authors $[2,3]$ 
have been developing a novel, unstructured S-F technique which allows to relieve much of the algorithmic complexity that plagued the "traditional" S-F techniques developed in the structuredgrid framework. This paper describes a recently developed version of the unstructured S-F algorithm, which allows to compute compressible viscous flows featuring SWBLIs; the results obtained using both S-C and S-F simulations on nearly identical triangular meshes have been compared to the experimental data in order to highlight the differences between the two shockmodeling approaches.

\section{SHOCK-FITTING ALGORITHM}

The present S-F technique for unstructured grids was first published by two of the authors in [3] and later improved and enhanced in $[4,5,6]$. Even though the technique has already been used $[7,8]$ to simulate viscous flows, those flow-cases did not involve SWBLIs, which require the additional algorithmic ingredients described here and in a recent, previous publication [11].

The present paper completes the description of the algorithmic developments and broadens the SWBLIs considered. The S-F algorithm relies on the use of a background triangular mesh, fixed in space, which covers the entire two-dimensional computational domain, and a onedimensional shock-mesh, which describes the moving discontinuity. As shown in the sequence of frames of Fig. 1, these two geometrical discretizations are merged to form what we call the "computational" mesh. More precisely, Fig. 1a is the "background" mesh, which will also be used to perform all the S-C calculations presented in Sect. 3. The shock-mesh, which is shown

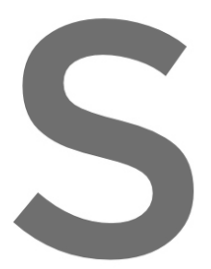

in all frames of Fig. using a red line is made up of an ordered sequence of shock-points. which are mutually connected using straight shock-edges. Both the shock-points and shock-
edges are duplicated items that share the same geometrical location: the shock-upstream and
shock-downstream flow states along the shock-mesh are stored within each pajr of shock-points
and, by also duplicating the shock-edges, the fitted-shock-behaves tike an ipterior boundary of
zero thickness. Observe from Fig. 1a that the geometrical location of the shock-points is totally
un-related to the location of the grid-points of the background triangulation. Let us now assume


pairs of shock-points. The algorithm that updates the field solution and the shock-states and position at time $t+\Delta t$ consists in ifve steps, which will be described hereafter.

\subsection{Step 1: cell removal around the shock front}

The first step consists in removing those triangles of the background mesh which are crossed by the discontinuity, and also the triangular cells that have at least one of their vertices that is too close to the shock-front. By doing so, a mesh-less hole, which contains the discontinuity, is carved within the background mesh, as shown in Fig. 1b.

\subsection{Step 2: local re-meshing around the shock front}

The hole is separately re-meshed using a constrained Delaunay triangulation (CDT), see Fig. 1c: the shock-edges and the boundaries of the hole are constrained to belong to the final CDT and no further grid-point is added. Re-meshing is localized around the discontinuity and, therefore, it has a limited computational cost. 


\subsection{Step 3: grid assembly}

The "computational" mesh that will be used to advance the solution in time from $t$ to $t+\Delta t$ is made up of the two different grids that have been generated in the previous two steps, see Fig. 1d: the background mesh with the hole and the CDT that fills the hole. When merging these two meshes, it is important to remove the duplicated nodes and ensure a consistent numbering of both the grid-points and the triangular cells.

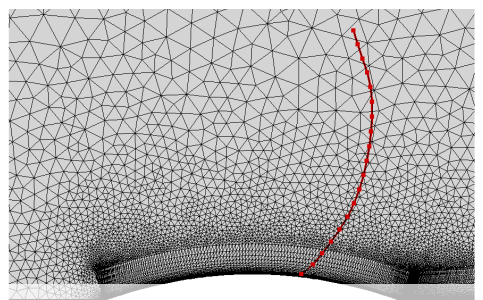

(a) Background mesh and shock at time t

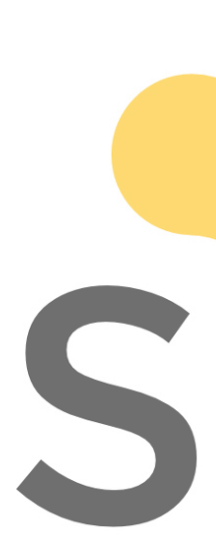

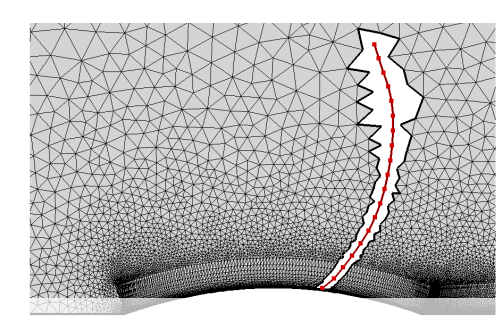

(b) Generation of the hole in the background mesh

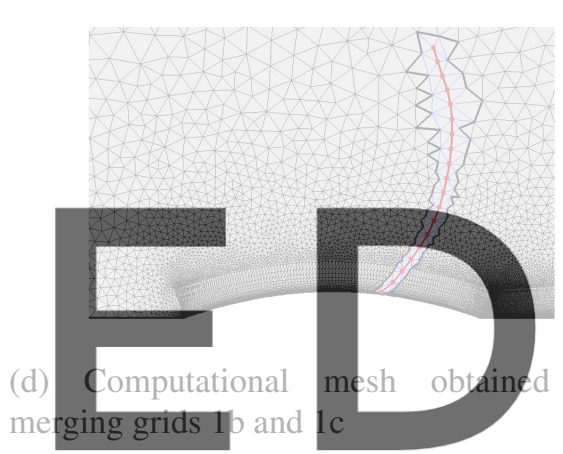

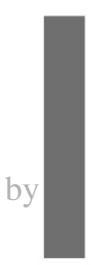

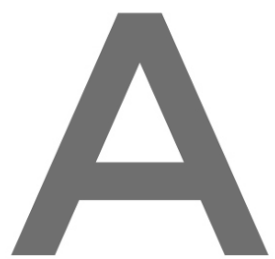

Figure 1: Sequence of steps for the generation of the computational mesh at time $t$

Register for free at https//wWw.scipedia.com to download the version without the watermark

\subsection{Step 4: calculation of the tangent and normal unit vectors to the shock front}

The tangent and normal unit vectors along the shock-front are needed within each shock-point in order to apply the R-H relations (step 5). These unit vectors are computed using finitedifference (FD) formulae which involve the coordinates of the shock-point itself and those of its neighboring shock-points. As described in [3], the FD stencil, either upwind or centered, is chosen depending on the local shock-downstream flow regime. Moreover, the presence of the boundary layer requires special provisions to be made concerning the calculation of the normal vector within the shock-point closer to the wall. This is because in viscous flows the no-slip condition prevents the shock from reaching the wall, which implies that it is not any longer possible to use the wall geometry to compute the shock-slope, as one would do when dealing with inviscid flows, which can be supersonic all the way down to the wall. For instance, this is the situation that occurs when dealing with the transonic turbulent flow past an airfoil, a test-case that will be extensively investigated and discussed in Sect. 3.2. Figure 2a shows the computed Mach-number field which is characterized by the presence of a supersonic pocket bounded on its downstream side by a shock-wave, shown using a black solid line in Fig. 2. As shown in Fig. 2b, 
which shows a zoom centered around the foot of the shock, the shock originates at a certain distance above the wall, due to the coalescence of compression waves. Since the intensity of the shock wave in the first shock-point (SP) is certainly very small, it can be assumed, as done in [9], that the local shock-slope coincides with that of the local Mach line; therefore, the vector normal to the shock can be taken perpendicular to the Mach line, as shown in Fig. 2 b.

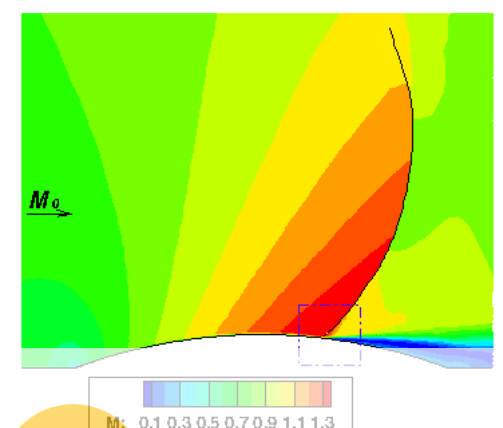

(a) Mach flood computed using S-F technique. The fitted shock is drawn using a black solid line.



(b) Enlargement around the foot of the shock: the shock originates due to the coalescence of characteristic curves, drawn in blue.

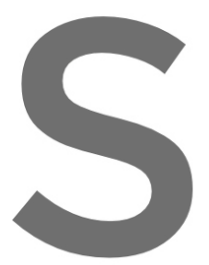

Figure 2: Transoniq the wall.

\section{5}

\section{Using the computational grid as input, a sing}
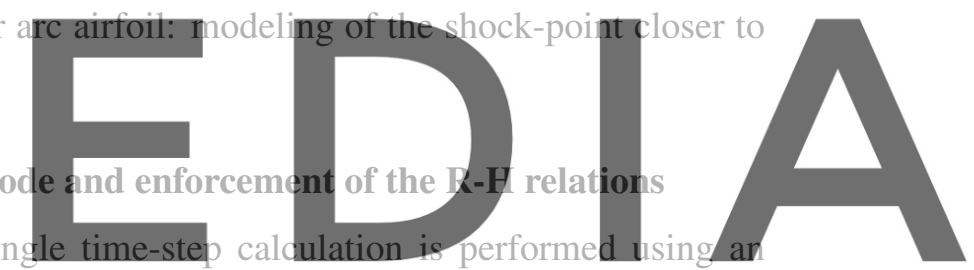

unstructured, vertex-centered, S-C solver eulfs [10], which returns updated nodal values at time

Register for freet at

of the shock need to be corrected by enforcing the R-H relations across each pair of shock-points:

this amounts to solve a system of non-linear algebraic equations which also supplies the local

shock-speed, as described in detail in [3]. Finally, knowledge of the shock-speed within all pairs of shock-points allows to move the shock-mesh from its current location to the updated one at time $t+\Delta t$ so that solution can be advanced over the next time-interval by repeating steps 1 to 5 .

\section{NUMERICAL RESULTS}

SWBLIs turn out to be an important test-bench to evaluate the capability of the proposed unstructured S-F technique to deal with both laminar and turbulent flows featuring SWBLIs. The SWBLI patterns that are more commonly encountered in nature are the following two. The first one consists in the coalescence of compression waves within the supersonic part of the boundary layer, leading to the formation of a shock-wave. The second one consists in a weak oblique shock-wave which penetrates the supersonic boundary layer, gradually weakening until it completely disappears when it reaches the sonic line. The first patterns occurs in the regular reflection of an impinging shock, which will be addressed in Sect. 3.1, whereas the latter occurs 
when dealing with shock formation over a transonic airfoil, and will be addressed in Sect. 3.2. In addition to a qualitative comparison between the two shock-modeling options, the computed results will be compared against the available experimental data and a quantitative assessment of the order-of-convergence of the two techniques will be made, using Richardson Extrapolation (RE) techniques.

\subsection{Regular oblique-shock reflection}

The interaction between an oblique shock and a boundary-layer is termed "strong" whenever it causes boundary-layer separation, see Fig. 3a. This is the case of the experimental and numerical study conducted by Degrez et al. [12], whose free-stream flow conditions are reported in Tab. 1 .

\begin{tabular}{|c|c|}
\hline Parameter & Value \\
\hline Mach & 2.2 \\
\hline Re $_{\infty} / \mathrm{m}$ & $1.210^{6}$ \\
\hline Total temperature & $293 \mathrm{~K}$ \\
\hline
\end{tabular}

Table 1: Oblique-shock reflection: free-stream conditions.

A sketch of the flow pattern and the computational domain used for reproducing the experimental conditions are shown in Fig. 3a and 3b, respectively. The incident shock of Fig. 3a, which forms because of the flow-defiection-imparted by the shock generator in Fig. 3b, erosses the

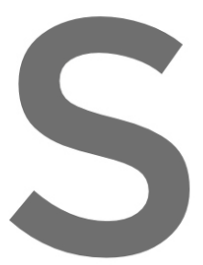
leading edge (L.E.) sh the lower wall. The boundary-layer separa
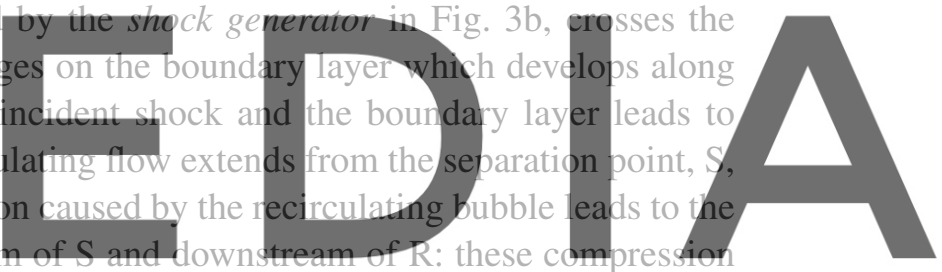

waves coalesce at some distance from the flat plate to form the separation and re-attachment Register for fireekat https//www.scipedia.com to download the version without the watermark

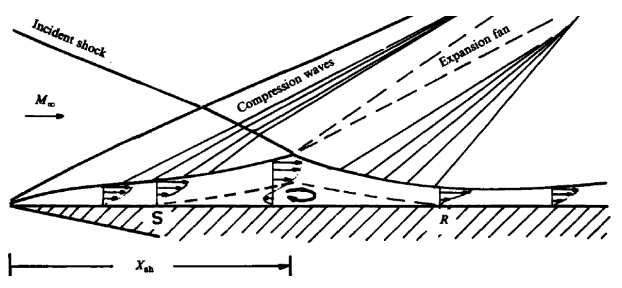

(a) Schematic of the flow-field due to SWBLI. Reprinted from [12], with permission.

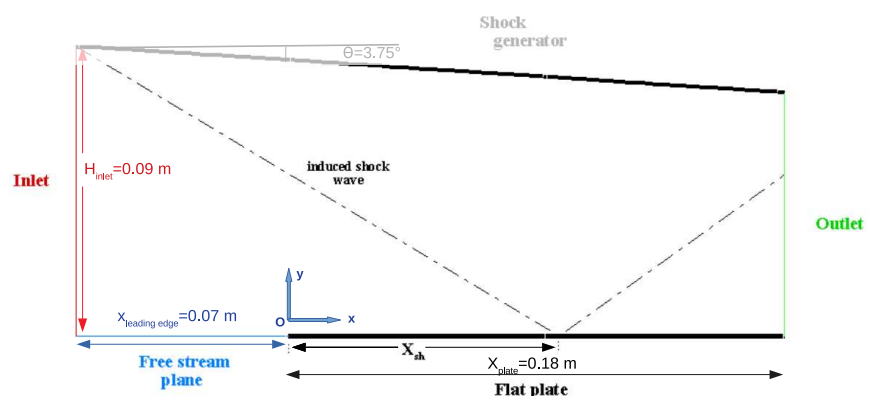

(b) Computational domain geometry.

Figure 3: Oblique-shock reflection: flow-field sketch and computational domain description.

In order to compare the grid-convergence properties of the S-C and S-F solutions, three nested 
grid levels have been obtained by recursively coarsening the finest one; their characteristic are summarized in Tab. 2. The unstructured triangular grids have been obtained from structured ones by dividing each quadrilateral cell into two rectangular triangles. S-C calculations have been performed using the eulfs code and the same background meshes also used in the S-F calculation. The comparison between Fig. 1a (the background mesh) and Fig. 1d (the computational mesh) clearly reveals that the S-C and S-F simulations have been performed using nearly identical grids: differences between the two are limited to the region close to the fitted shock, where local re-meshing has been used to accommodate the shock-mesh inside the background mesh. These differences are quantitatively summarized in Tab. 2, where the percentage increase in the grid-point and cell count is also provided for all three S-F grids. On each grid level the S-C calculations have been run first. Then, a shock-detection algorithm, such as the one described in [13], has been used to extract from the S-C calculation the approximate shape and location of the fitted discontinuities; the fitted discontinuities and the flow solution computed by means of S-C supply the initial condition to the S-F calculations. The S-F technique has been used in an hybrid manner: the discontinuities have been fitted, but their mutual interaction has been captured.
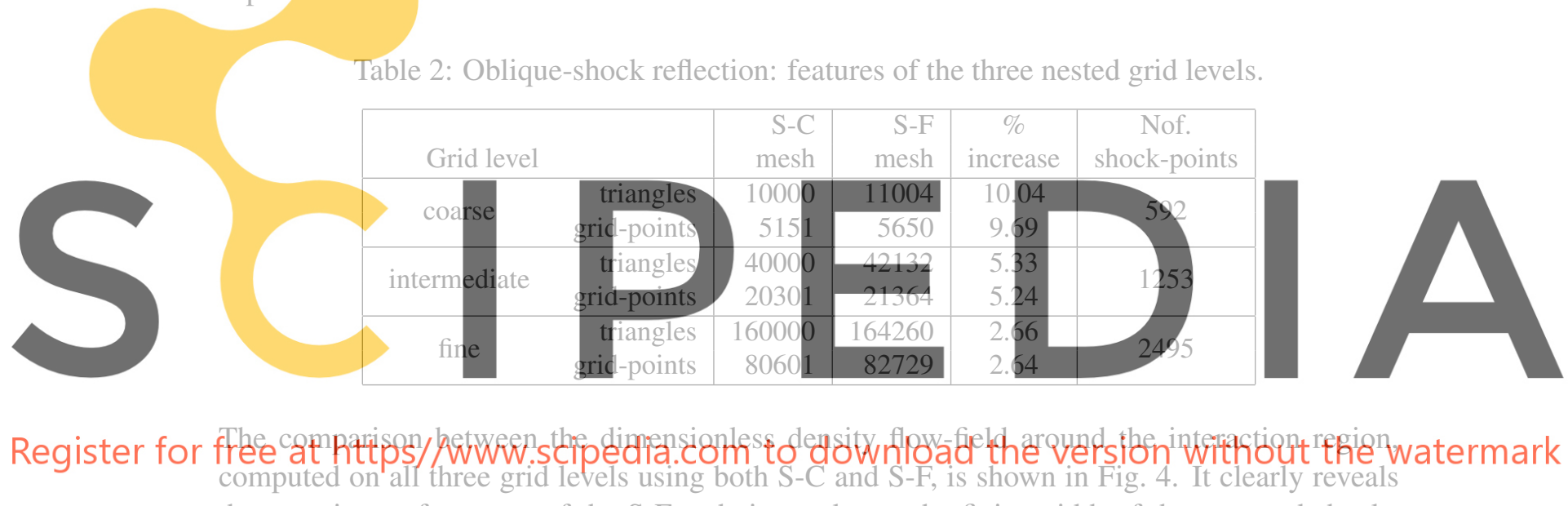
the superior performance of the S-F technique: due to the finite width of the captured shocks the interaction region is poorly solved, particularly on the coarse grid, see Fig. 4a. When using S-C, the incident shock can be clearly identified only on the finest grid level, Fig. 4f, whereas the $\mathrm{S}-\mathrm{F}$ technique represents the incident shock (drawn using a white solid line in Figs. 4b, 4d and 4f) as a true discontinuity on all grid levels. A preliminary and qualitative grid-convergence study has been performed by analyzing both the skin friction (Fig. 5) and wall pressure (Fig. 6) distributions computed on all grid levels using the two different shockmodeling options. Figures 5a and 6 a respectively show the skin-friction and wall-pressure distributions computed using S-C on all three grid levels, whereas the S-F computed results are displayed in Figs. 5b and 6b. When looking at the S-C and S-F calculations, both are seen to converge towards the same fine-grid solution as the mesh is refined, but S-F appears to be converging faster. For instance, the S-F simulation already reveals on the coarsest grid level the skin friction and pressure plateau within the separation region, whereas the same feature becomes visible on the intermediate grid level using the $\mathrm{S}-\mathrm{C}$ approach. As far as the wall pressure distribution is concerned, the agreement between the two shock-modeling options, and 
also with the experimental data available in [12], is good.

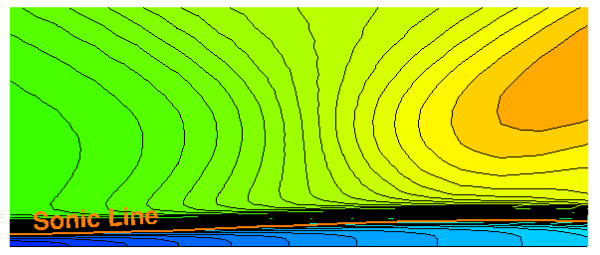

(a) S-C computation: coarse grid.

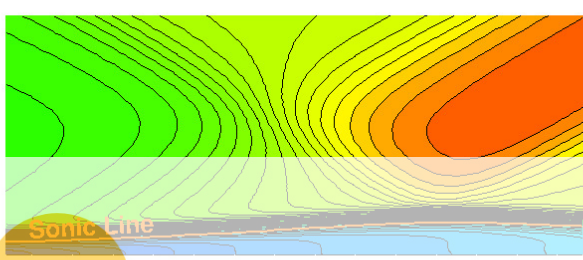

(c) S-C computation: intermediate grid.

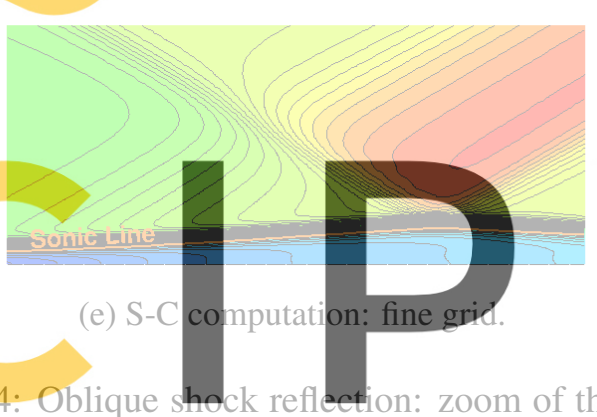

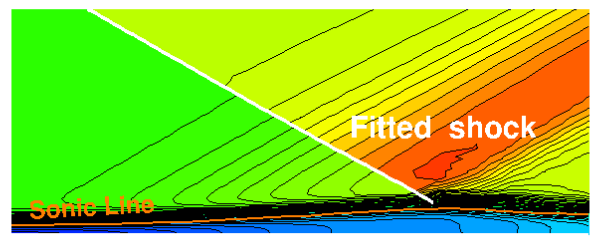

(b) S-F computation: coarse grid.

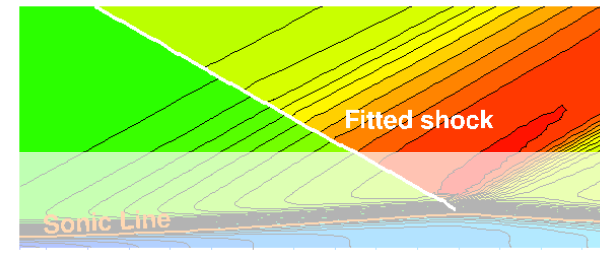

(d) S-F computation: intermediate grid.

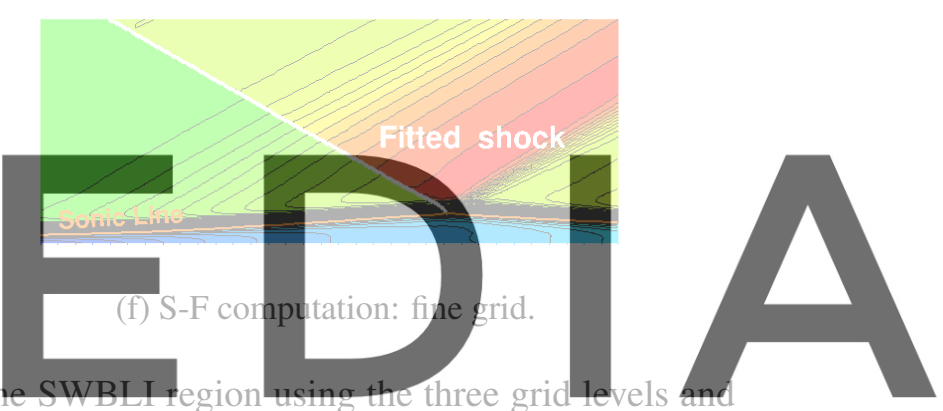

Figure 4: Oblique shock
shock-modeling options.

Register for free at https//www.scipedia.com to download the version without the watermark

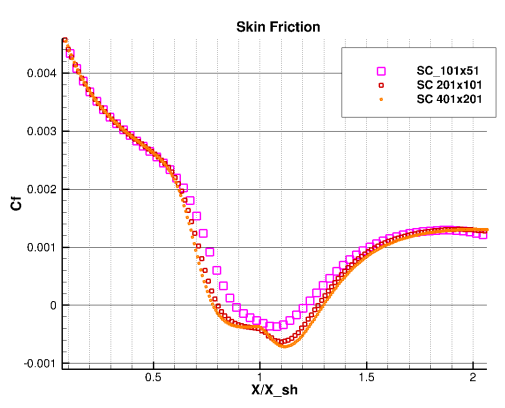

(a) S-C on all grid levels.

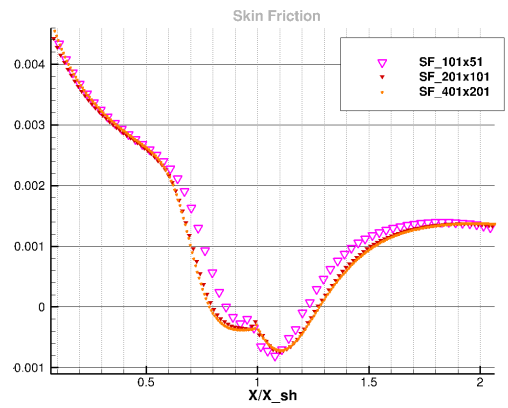

(b) S-F on all grid levels.

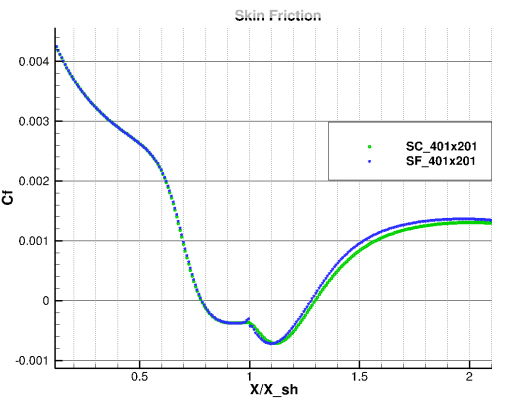

(c) S-C vs. S-F on the finest grid.

Figure 5: Oblique-shock reflection: skin friction distribution. 




(a) S-C on all grid levels.

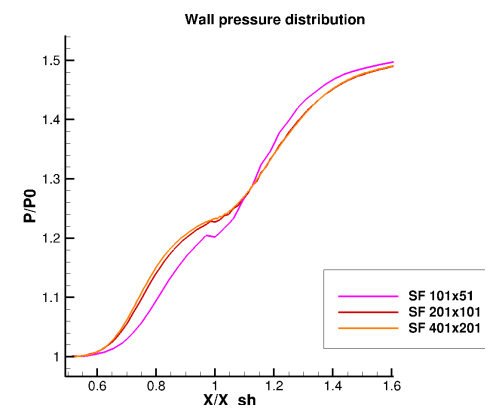

(b) S-F on all grid levels.

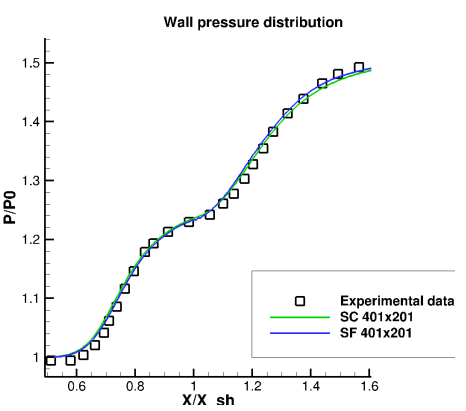

(c) S-C vs. S-F on the finest grid.

Figure 6: Oblique-shock reflection: wall pressure distribution.

A quantitative assessment of the grid-convergence properties of the two different shock-modeling options has been conducted using Richardson Extrapolation (RE), and it is presented in Tab. 3, which shows the location of the separation and re-attachment points (their abscissae have been measured relative to the L.E. and have been normalized by the distance, $\mathrm{X}_{s h}=80 \mathrm{~mm}$ ) and the size of the recirculation bubble computed using both modeling options on all three mesh levels. The observed order of accuracy, $\tilde{n}$, is calculated as follows:
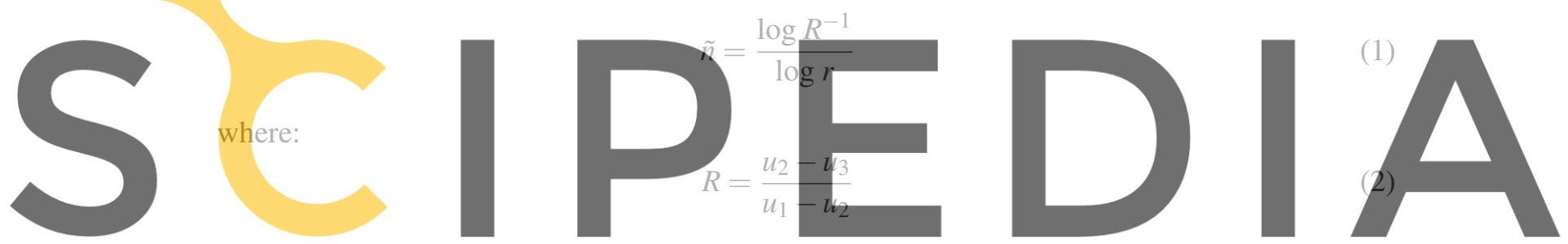

is the so-called convergence monitor and the grid-refinement-ratio, $r$, is constant and equal to

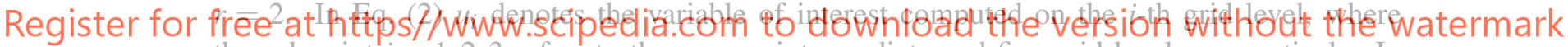
the subscript $i=1,2,3$ refers to the coarse, intermediate and fine grid levels, respectively. In

Table 3: Oblique-shock reflection: grid-convergence study and RE in the separation region.

\begin{tabular}{|c|c|c|c|c|c|c|}
\hline \multicolumn{5}{|c|}{ Shock capturing } & \multicolumn{3}{c|}{ Shock fitting } \\
\hline Grid & $\left(\mathrm{X}_{\text {sep }} / \mathrm{X}_{\text {sh }}\right)$ & $\left(\mathrm{X}_{r e} / \mathrm{X}_{s h}\right)$ & Bubble size $(\mathrm{cm})$ & $\left(\mathrm{X}_{\text {sep }} / \mathrm{X}_{\text {sh }}\right)$ & $\left(\mathrm{X}_{r e} / \mathrm{X}_{\text {sh }}\right)$ & Bubble size $(\mathrm{cm})$ \\
\hline coarse & $0.87 \pm(0.13)$ & $1.22 \pm(0.12)$ & $2.73 \pm(1.98)$ & $0.86 \pm(0.11)$ & $1.21 \pm(0.09)$ & $2.78 \pm(1.58)$ \\
intermediate & $0.80 \pm(0.04)$ & $1.27 \pm(0.05)$ & $3.77 \pm(0.67)$ & $0.80 \pm(0.03)$ & $1.26 \pm(0.02)$ & $3.71 \pm(0.42)$ \\
fine & $0.78 \pm(0.01)$ & $1.30 \pm(0.02)$ & $4.13 \pm(0.23)$ & $0.78 \pm(0.01)$ & $1.28 \pm(0.01)$ & $3.96 \pm(0.11)$ \\
\hline$\tilde{n}$ & 1.79 & 1.30 & 1.55 & 1.86 & 1.99 & 1.92 \\
\hline experimental values & $0.78 \pm(0.01)$ & $1.28 \pm(0.02)$ & $4.00 \pm(0.3)$ & $0.78 \pm(0.01)$ & $1.28 \pm(0.02)$ & $4.00 \pm(0.3)$ \\
\hline
\end{tabular}

particular, the observed order-of-accuracy of the S-F solutions is very close to the design order $(n=2)$ of the discretization scheme, which is an indication $[14,15]$ that the S-F solutions are in the asymptotic range of convergence. The analysis can be further expanded by computing the Grid Convergence Index (GCI) [14]. On the $i$-th grid level:

$$
\mathrm{GCI}_{i}=1.25\left|u_{i}-\tilde{u}_{R E}\right|
$$


where the same notation of Eqs. (1) and (2) has been used and:

$$
\tilde{u}_{R E}=u_{3}-\frac{u_{2}-u_{3}}{r^{\tilde{n}}-1} .
$$

is the extrapolated value. The GCI is a measure of the numerical error, just like the experimental uncertainty; GCI values of the bubble size, separation and re-attachment locations are reported between brackets alongside each numerical value in Tab. 3, whereas bracketed values beside the measured data refer to the experimental uncertainty [12]. Observe that almost all GCI values are smaller for the S-F solutions than the corresponding ones for S-C, highlighting a faster convergence of the former technique over the latter.

\subsection{Transonic, turbulent flow past an airfoil}

We have numerically reproduced a test-case which has been experimentally studied by McDevitt et al. in [17], using the free-stream conditions reported in Tab. 4. It consists in the transonic flow over a $18 \%$-thick biconvex circular-arc airfoil characterized by a SWBLI leading to fully turbulent, separated flow. Turbulence closure of the compressible, Reynolds averaged NavierStokes (RANS) equations relies on the one-equation Spalart-Allmaras model (S-A) [16].

Table 4: Transonic, turbulent flow past an airfoil: free-stream flow conditions.
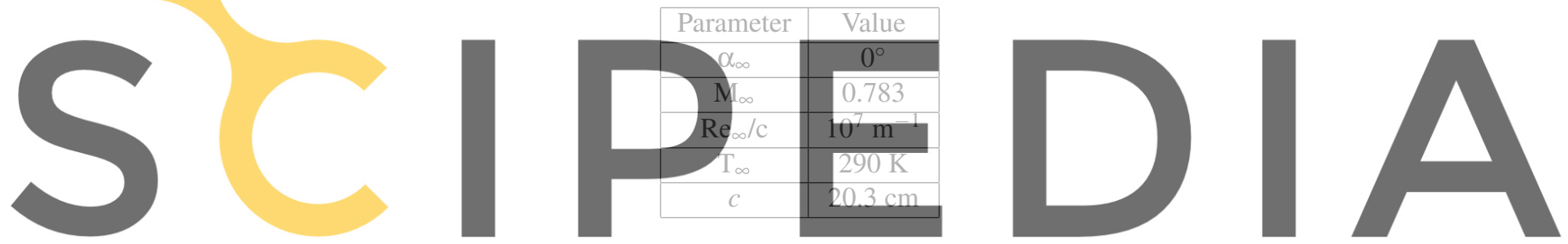

The computational domain is displayed in Fig. 7, where all lengths have been made dimensionless

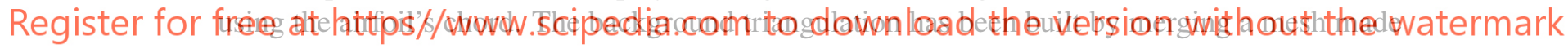
of rectangular triangles, which covers the near-wall and wake regions, with a fully unstructured triangulation. Exponential mesh-stretching has been used in the cross-flow direction to ensure adequate resolution both within the boundary layer and the wake.

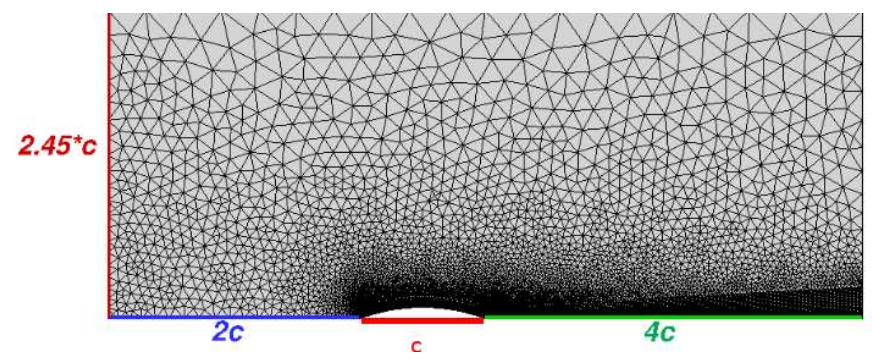

Figure 7: Transonic, turbulent flow past an airfoil: background mesh.

The flow-field is characterized by the presence of a supersonic pocket on the airfoil surface, bounded by the sonic line on its upstream side and within the boundary layer and closed by a 
shock-wave on its downstream side. Figures $8 \mathrm{a}$ and $9 \mathrm{a}$ show the density flow-field computed using S-C and S-F, respectively. The enlarged views of Figs. $8 \mathrm{~b}$ and $9 \mathrm{~b}$ again point to the very different shock-thickness delivered by the two different shock-modeling options. A more quantitative comparison between the S-C and S-F calculations is shown in Fig. 10, where the pressure coefficient distribution is plotted along the airfoil's surface. Also shown are the experimental data measured by McDevitt et al. [17]. The two different shock-modeling options show nearly identical distributions of the pressure coefficient, both of which are in fair agreement with the experimental data. A visible difference between the experimental and computational data has to do with the location of the shock wave, which is predicted a little bit downstream of the experimental location in both the S-C and S-F simulations. The origin of the discrepancy between simulations and experiments appears to be rooted in the turbulence model being used. This has been assessed by running two additional S-C calculations on the background mesh using the CFD++ commercial software and both the S-A and $k-\varepsilon$ models. These results have also been plot in Fig. 10 and show that the CFD++ calculation with the S-A model predicts the same shock position also found in our calculations, whereas the $k-\varepsilon$ turbulence model locates the shock even further downstream.
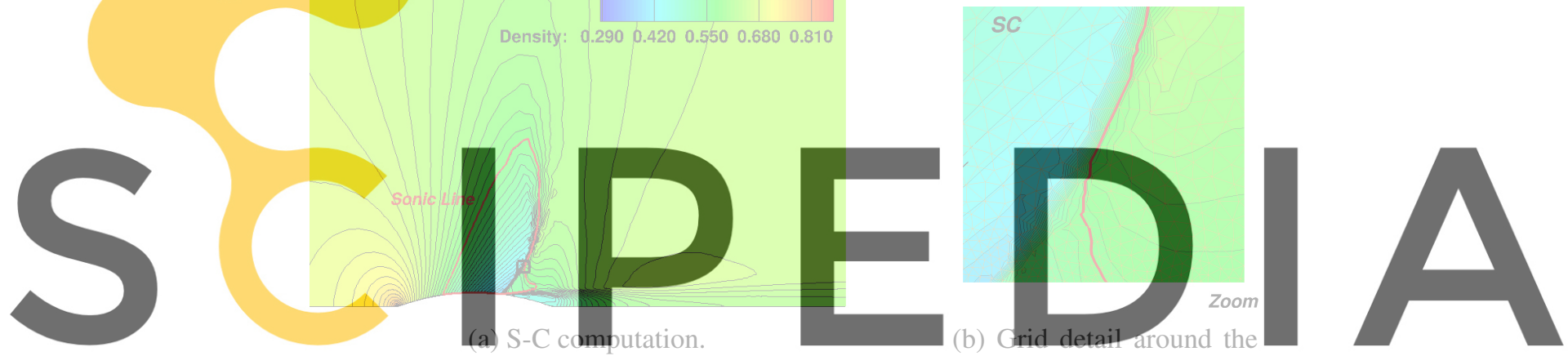

Register for free at https//www.scipedia.com to download
Figure 8: Transonic, tufbulent flow past an airfoil: S-C

captured shock

the version without the watermark omputation and grid detail

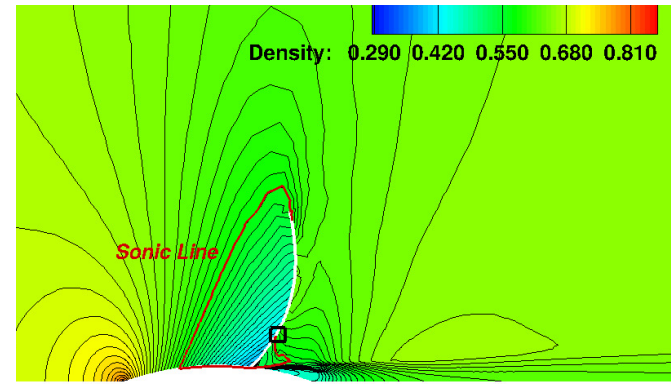

(a) S-F computation: the fitted shock is shown using a white line.

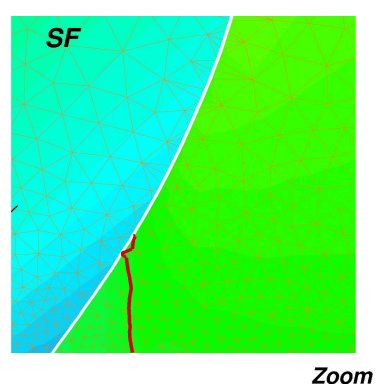

(b) Local re-meshing detail around the fitted shock

Figure 9: Transonic, turbulent flow past an airfoil: S-F computation and grid detail 


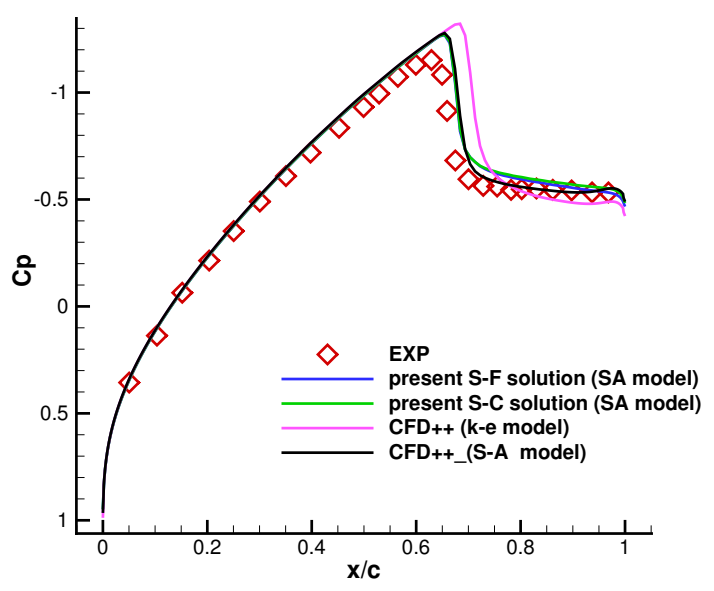

Figure 10: Transonic, turbulent flow past an airfoil: pressure coefficient distribution

\section{CONCLUSIONS}

An unstructured, shock-fitting technique developed by the authors has been successfully extended to laminar and turbulent viscous flows featuring shock-wave/boundary-layer interactions. Comparisons have been made between the S-C and the S-F modeling approaches: computed results show that S-F can significantly improve the quality of the solution and the agreement with the experimental data when compared to a S-C calculation computed on nearly identical meshes. Moreover, a grid-convergence analysis reveals that S-F solutions feature a measured order-of-convergence which is closer to design order than that observed in the S-C calculations. This is tantamount to saying that a given discretization error can be obtained using S-F on (much) coarser meshes than those required by S-C. This is of utmost importance for viscous flow numerical simulations, which are computationally expensive, even more in three spatial dimensions. Therefore, future work includes the extension of the algorithm to three-dimensional flows characterized by SWBLIs.

\section{REFERENCES}

[1] Moretti G., Abbett M., A time-dependent computational method for blunt body flows, AIAA Journal (1966) ,pp. 2136-2141.

[2] Paciorri R., Onofri M., Shock Fitting: classical technique,recent developments and memoirs of Gino Moretti. Springer International Publishing, 2017.

[3] Paciorri R., Bonfiglioli A., A shock-fitting technique for 2D unstructured grids, Computers and Fluids, 38(3),pp. 715-726, 2009.

[4] Paciorri R., Bonfiglioli A., Shock interaction computations on unstructured, twodimensional grids using a shock-fitting technique, Journal of Computational Physics, 230(8),pp. 3155-3177, 2011.

[5] A. Bonfiglioli, M. Grottadaurea, R. Paciorri, F. Sabetta, An unstructured, threedimensional, shock-fitting solver for hypersonic flows, Computers and Fluids, 73,pp. 162174, 2013. 
[6] Paciorri R., Bonfiglioli A., Campoli L., Unsteady shock-fitting for unstructured grids, International Journal for Numerical Methods in Fluids, 81(4), 245-261, 2016.

[7] A. Bonfiglioli, R. Paciorri, Comparative study of stagnation point anomalies by means of shock capturing and shock fitting unstructured codes,Proceeding of The 6th European Symposium on Aerothermodynamics for Space Vehicles, 3-6 November 2008, Versailles, France, Vol. 659 SP, European Space Agency, ESA, (2009).

[8] Lani A., De Amicis V., Shock Fitting: An Open Source Object-Oriented Platform for Unstructured Shock-Fitting Methods In: Onofri M., Paciorri R., (eds.) Shock Fitting: Classical Techniques, Recent Developments, and Memoirs of Gino Moretti, Springer International Publishing(2017), pp. 85-112.

[9] G. Moretti, Circumspect exploration of multidimensional imbedded shocks. AIAA Journal 14(7), pp. 894-899, 1976.

[10] Bonfiglioli A., Fluctuation splitting schemes for the compressible and incompressible Euler and Navier-Stokes equations. International Journal of Computational Fluid Dynamics 14 (2000),pp. 21-39.

[11] Assonitis A., Paciorri R., Bonfiglioli A. Numerical Simulation of Shock Boundary Layer Interaction Using Shock Fitting Technique. Proceedings of XXIV AIMETA Conference 2019. AIMETA 2019. Lecture Notes in Mechanical Engineering. (2020)

[12] G. Degrez, C. H. Boccadoro, J. F. Wendt, The interaction of an oblique shock wave with a laminar boundary layer revisited. an experimental and numerical study, Journal of Fluid Mechanics 177 (1987) 247-263.

[13] Paciorri R., Bonfiglioli A., Accurate detection of shock waves and shock interactions in two-dimensional shock-capturing solutions, Journal of Computational Physics 06 (2020)

[14] Roache P. J., Quantification of uncertainty in computational fluid dynamics, Annual Review of Fluid Mechanics 29 (1) (1997) 123-160.

[15] A. Di Mascio, R. Paciorri, B. Favini, Truncation Error Analysis in Turbulent Boundary Layers , Journal of Fluids Engineering 124 (3) (2002) 657-663. doi:10.1115/1.1478564

[16] Spalart P., Allmaras S., A one-equation turbulence model for aerodynamic flows, $L a$ Recherche-Aerospatiale 1 (1994) 5-21.

[17] McDevitt J. B., Supercritical flow about a thick circular-arc airfoil, Tech.rep., NASA Ames Research Center (01 1979) 